\title{
Comparaison de différentes approches pour la simulation numérique d'impacts hydrodynamiques
}

\author{
Nicolas Jacques* - Adrian Constantinescu** \\ Steven Kerampran* — Alain Nême* \\ * Laboratoire Brestois de Mécanique et des Systèmes, ENSIETA \\ Université de Bretagne Occidentale/ENIB \\ 2 rue François Verny, 29806 Brest cedex 9, France \\ \{nicolas.jacques, steven.kerampran,nemeal\}@ensieta.fr \\ ** ANAST, Université de Liège \\ Chemin des Chevreuils 1, 4000 Liège, Belgique \\ A.Constantinescu@ulg.ac.be
}

RÉSUMÉ. Le but de ce travail est de comparer différentes méthodes numériques appliquées à la simulation d'impacts hydrodynamiques. Trois modèles ont été considérés : (i) un modèle éléments finis basé sur la théorie de Wagner, (ii) un modèle éléments finis basé sur un formalisme Arbitraire Lagrange-Euler (ALE), (iii) un modèle volumes finis basé sur un formalisme eulérien multiphasique et la technique "Volume of Fluid 》. Les capacités de ces différentes approches à résoudre des problèmes d'impact hydrodynamique sont étudiées au travers de comparaison avec des résultats de référence de la littérature et avec des résultats expérimentaux.

ABSTRACT. The aim of this paper is to compare several simulation methods for hydrodynamic impact problems. For this purpose, three models has been developed: (i) a finite element model based on the Wagner theory, (ii) a finite element model based on a Arbitrary Lagrangian Eulerian (ALE) formulation, (iii) a finite volume model based on a two-phase eulerian formalism and the Volume-of-Fluid technique. The different results are compared with published and experimental data.

MOTS-CLÉS: impacts hydrodynamiques, théorie de Wagner, formalisme ALE, formalisme eulérien multiphasique.

KEYWORDS: hydrodynamic impact problems, Wagner theory, ALE formulation, multi-phase eulerian formulation.

DOI:10.3166/EJCM.19.743-770 C 2010 Lavoisier, Paris 


\section{Introduction}

L'impact hydrodynamique désigne le choc d'une structure sur la surface d'un fluide faiblement compressible (par exemple de l'eau). Ce phénomène survient par exemple lorsqu'un navire rencontre des conditions de mer difficiles. En effet, différents facteurs comme la vitesse du navire et la hauteur de la houle peuvent amener la carène à émerger partiellement puis à retomber violemment sur la surface de l'eau. Ce phénomène, appelé tossage ou slamming dans le domaine naval, peut avoir des conséquences très néfastes sur l'intégrité structurale des bâtiments de surface. En effet, les efforts induits par le tossage sont actuellement les plus importants que subissent les navires, en dehors d'agressions accidentelles. Une fréquence trop importante des impacts peut conduire à une fatigue prématurée de la structure. Dans ce contexte, on comprend l'importance pour l'architecte naval de connaître les efforts induits par le tossage. Un des premiers travaux sur ce sujet a été publié par von Kármán (1929). En utilisant la notion de masse ajoutée, ce dernier a proposé une formule analytique très simple qui permet d'estimer l'effort de choc lors de l'impact d'un dièdre. Wagner (1931) a proposé également un modèle analytique pour l'impact de dièdres. Dans ce travail, l'écoulement autour du corps impactant est estimé à l'aide de la théorie du potentiel. Un élément essentiel du modèle proposé par Wagner est la linéarisation du problème fluide par projection des conditions aux limites sur la surface initiale (donc plane) du domaine fluide (figure 1). On comprend que cette hypothèse limite la validité de la théorie de Wagner aux cas où les angles de pénétration sont relativement faibles. Ses travaux ont été ensuite repris et améliorés par de nombreux chercheurs. Des solutions analytiques sont, par exemple, disponibles dans le cas de corps impactants tridimensionnels (Korobkin, 2002) (avec néanmoins des restrictions importantes sur la forme) et déformables (couplage hydro-élastique) (Scolan, 2004 ; Khabakhpasheva, 2009). De plus, des travaux ont été menés pour étendre la théorie de Wagner à des angles d'incidence plus importants (Cointe, 1989; Korobkin, 2004). Il existe par exemple ce qu'on appelle la théorie de Wagner généralisée, où seules les conditions aux limites au niveau de la surface libre sont projetées sur une surface plane (Mei et al., 1999 ; Faltinsen, 2002).

Néanmoins, si la qualité des prévisions des modèles analytiques a été vérifiée dans le cas de géométries simples, l'étude de structures complexes nécessite le recours à la simulation numérique. Dans ce cadre, de nombreuses approches sont possibles. Dans (Donguy, 2002 ; Peseux et al., 2005 ; Constantinescu, 2006) et (Constantinescu et al., 2008), des modèles numériques basés sur la théorie de Wagner sont proposés pour les cas bidimensionnels. Le problème potentiel linéarisé est, dans ce cas, résolu par la méthode des éléments finis. Cela permet de considérer des corps impactants rigides ou déformables de forme complexe. Néanmoins, la validité de ces modèles reste bien sûr limitée aux cas des angles d'incidences modérés. Pour traiter des angles d'incidence plus grands, (Zhao et Faltinsen, 1993), (Battistin et Iafrati, 2003), (Kim et Shin, 2003) et (Sun et Faltinsen, 2006) ont proposé des modèles basés sur la théorie du potentiel non linéaire. Dans ces travaux, la solution est obtenue à l'aide de la méthode des éléments de frontière. La simulation du slamming a également été traitée à l'aide de 
codes plus généralistes basés sur un formalisme lagrangien ou arbitraire LagrangeEuler (ALE). Par exemple, Ribet et al. (1999) ont simulé l'impact de structures élastiques sur l'eau à l'aide du code de calcul par éléments finis Radioss. L'approche ALE a également été utilisée par Federico et Amoruso (2009) pour étudier l'impact plan d'un solide rigide sur la surface d'un fluide au repos, ainsi que l'impact de débris fluides sur la surface d'un solide. Une limitation de cette approche est qu'elle ne permet pas de simuler d'écoulements fragmentés. De plus, sa mise en œuvre peut être délicate à cause de problèmes liés à la distorsion du maillage. Une autre approche possible est l'utilisation de codes basés sur un formalisme eulérien multiphasique utilisant une technique de capture d'interface telle que la méthode VOF, (Varyani et al., 2000 ; Azcueta, 2001 ; Kleefsman et al., 2005 ; Aquelet et al., 2006 ; Leroyer et al., 2007) ou Level-Set (Colicchio et al., 2005). Dans ce cas, des écoulements complexes peuvent être simulés. Citons également les travaux de Colicchio et al. (2006), dans lesquels, la méthode Level-Set et celle des éléments de frontières sont utilisées conjointement grâce à l'utilisation d'une technique de décomposition de domaine. Récemment, des méthodes sans maillages ont été proposées pour la simulation de problèmes d'interaction fluide-structure (Rompteaux et Vila, 1999; Idelson et al., 2003 ; Oger et al., 2006; Gonzáles et al., 2007). Ces modèles sont basés sur un formalisme Lagrangien, mais les déformations extrêmes apparaissant au sein du fluide ne sont plus un problème.

Au vu de la diversité des approches existantes permettant la simulation d'impacts hydrodynamiques, on peut s'interroger sur la précision, la robustesse et la facilité de mise en œuvre de ces différentes méthodes. S'il existe de nombreuses publications portant sur la simulation numérique d'impacts hydrodynamiques, elles sont généralement focalisées sur une seule approche de modélisation. Et comme les applications traitées dans ces articles sont rarement les mêmes, il n'est pas facile en les lisant séparément de comparer ces méthodes et finalement de savoir dans quelle mesure elles sont adaptées au problème considéré. Dans le cadre de ce travail, qui est principalement destiné à des ingénieurs et des chercheurs ayant besoin de mettre en œuvre des simulations numériques pour analyser certains problèmes d'impacts hydrodynamiques, nous proposons d'évaluer les capacités de différentes méthodes numériques pour la modélisation du slamming. Trois approches ont été considérées :

1. modèle éléments finis basé sur la théorie de Wagner ;

2. modèle éléments finis basé sur un formalisme arbitraire Lagrange-Euler (ALE) ;

3. modèle volumes finis basé sur un formalisme eulérien multiphasique et la méthode « Volume of Fluid ».

Pour la réalisation des simulations avec le modèle 1, un outil spécifique dédié aux problèmes d'impact hydrodynamique a été développé. Les modèles 2 et 3 reposent quant à eux sur l'utilisation de codes commerciaux généralistes : ABAQUS/Explicit et Fluent, respectivement. Notons que les modèles 1 et 2 peuvent être utilisés pour traiter le cas de structures flexibles. Ce qui n'est pas le cas du 
modèle 3, qui nécessiterait pour cela d'être couplé à un outil de calcul de structures. Dans cette étude, des simulations bidimensionnelles ont été réalisées pour différentes formes de corps impactant. Les difficultés que nous avons rencontrées dans la mise au point de ces simulations, ainsi que les avantages des différents modèles, sont discutés. Les résultats obtenus sont ensuite comparés entre eux, ainsi qu'avec des résultats théoriques et de simulations issus de la littérature, et avec des données expérimentales. Au final, une bonne concordance entre les résultats des différentes approches est observée. Les résultats obtenus avec le modèle 1 sont parfois un peu différents. Cela est attribué aux hypothèses simplificatrices à la base de la théorie de Wagner sur laquelle est basé cet outil.

\section{Les modèles numériques utilisés}

\subsection{Modèle éléments finis basé sur la théorie de Wagner}

Dans cette section, nous présentons brièvement le code Impact++ (Constantinescu, 2006). Il s'agit d'un outil spécifique spécialement développé pour la simulation numérique d'impacts hydrodynamiques et qui s'appuie sur le formalisme proposé dans (Dongui, 2002) et (Peseux et al., 2005). La modélisation de l'écoulement du fluide est basée sur une théorie du potentiel linéarisée, appelée théorie de Wagner. Impact++ permet de traiter des problèmes bidimensionnels (plans et axisymétriques).

\subsubsection{Formulation du problème fluide}

Pour l'étude des problèmes d'impacts hydrodynamiques, il est généralement admis que les effets liés à la compressibilité et à la viscosité de l'eau, ainsi que ceux liés à la tension de surface, sont négligeables (Faltinsen, 2005). De plus, comme les accélérations subies par le fluide lors de l'impact sont généralement très fortes par rapport à celle de la gravité, cette dernière est également négligée. Avec ces hypothèses, les équations gouvernant l'écoulement du fluide sont :

$$
\begin{aligned}
& \operatorname{div} \vec{v}=0, \\
& \rho_{l}\left(\frac{\partial \vec{v}}{\partial t}+\vec{v} \cdot \nabla \vec{v}\right)=-\nabla p,
\end{aligned}
$$

où $\vec{v}$ désigne la vitesse du fluide, $p$ la pression hydrodynamique et $\rho_{l}$ la masse volumique du liquide.

Nous supposons également que le fluide est initialement au repos. D'après le théorème de Lagrange, l'écoulement sera donc irrotationnel et il est possible d'introduire une fonction scalaire $\Phi$ appelée potentiel des vitesses telle que :

$$
\vec{v}=\nabla \Phi \text {. }
$$


$\Phi$ est solution de l'équation de Laplace :

$$
\Delta \Phi=0 .
$$

En utilisant cette équation, ainsi que celle de la conservation de la quantité de mouvement [2], nous retrouvons l'équation de Bernoulli, qui permet de calculer la pression à partir du potentiel des vitesses :

$$
p=-\rho_{l}\left(\frac{\partial \Phi}{\partial t}+\frac{1}{2}(\nabla \Phi)^{2}\right) .
$$

Au niveau de la surface libre du fluide (figure 1a), la pression est nulle :

$$
\frac{\partial \Phi}{\partial t}+\frac{1}{2}(\nabla \Phi)^{2}=0 .
$$

Au niveau de l'interface entre le fluide et le solide, que nous appellerons par la suite surface mouillée, les composantes normales de la vitesse du fluide et du solide sont égales, d'où :

$$
\frac{\partial \Phi}{\partial n}=\vec{V}_{s} \cdot \vec{n}
$$

$\vec{V}_{s}$ est la vitesse du solide et $\vec{n}$ est la normale à la surface mouillée. Si l'on considère un domaine fluide infini, $\Phi$ doit également vérifier la condition suivante :

$$
\Phi \rightarrow 0 \quad \text { lorsque } \quad x^{2}+z^{2} \rightarrow 0
$$

Malgré les hypothèses que nous venons d'introduire, le problème fluide demeure difficile à résoudre. La principale difficulté est liée au fait que la surface du fluide va se déformer lors de l'impact et n'est donc pas connue. Afin de simplifier davantage le problème, nous allons maintenant introduire les hypothèses proposées par Wagner (1931) pour l'impact de corps avec de faibles angles de pénétration. Notons que c'est avec ce genre de géométries que les efforts d'impact sont les plus importants. Pour traiter ce cas, Wagner proposa de négliger la déformation de la surface du fluide et ainsi de projeter toutes les conditions aux limites sur la surface libre initiale (figure 1b). De plus, pour établir la condition de surface libre, le terme quadratique dans l'équation de Bernoulli [5] est également négligé. Avec ces hypothèses, et dans le cas d'un solide symétrique et convexe, le potentiel des vitesses vérifie dans le demi-plan inférieur $z<0$, les équations suivantes :

$$
\begin{cases}\Delta \Phi=0 & z \leq 0 \\ \Phi=0 & z=0 \text { et }|x|>d \\ \frac{\partial \Phi}{\partial z}=\vec{V}_{s} \cdot \vec{z} & z=0 \text { et }|x| \leq d \\ \Phi \rightarrow 0 & x^{2}+z^{2} \rightarrow 0\end{cases}
$$


Ce système d'équation est linéaire, mais une difficulté subsiste : la largeur de la surface mouillée $d$ n'est pas connue.
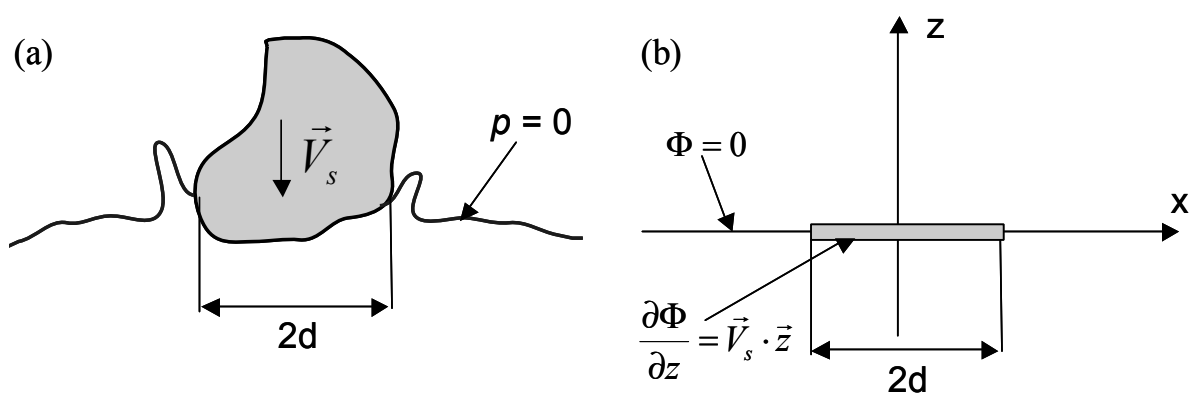

Figure 1. Illustration du problème d'impact hydrodynamique (a) et de son approximation dans le cadre de la théorie de Wagner (b)

\subsubsection{Détermination de la largeur mouillée}

La largeur mouillée $d(t)$ correspond à l'abscisse du point d'intersection entre la surface du solide impactant et la surface libre du fluide. $d(t)$ doit donc vérifier l'équation suivante :

$$
h(d(t), t)=f(d(t))+\vec{U}_{s}(t) \cdot \vec{z} .
$$

où $h(x, t)$ est l'élévation de la surface libre, $f(x)$ est la distance initiale entre le solide et la surface du liquide et $\vec{U}_{s}(t)$ est le déplacement du solide (figure 2).

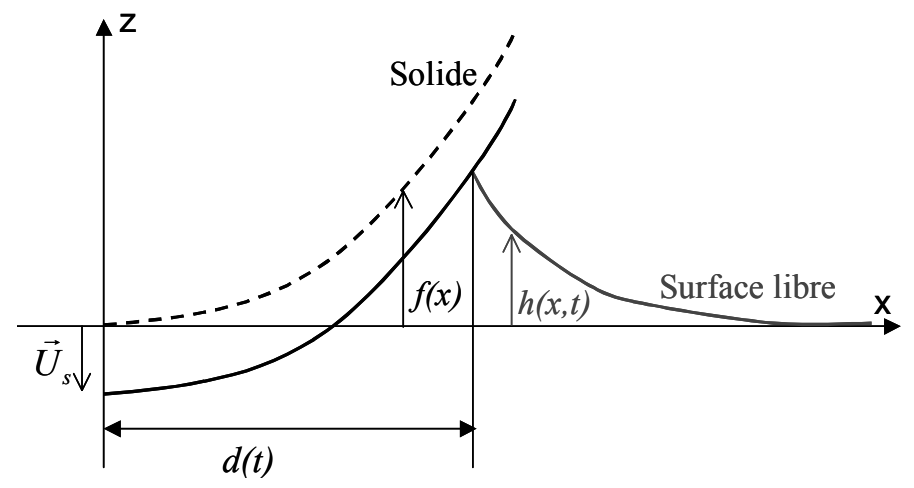

Figure 2. Définition de la largeur mouillée

Afin de calculer l'élévation de la surface libre, il est commode d'introduire le potentiel des déplacements : 


$$
\Psi(x, z, t)=\int_{0}^{t} \Phi(x, z, \tau) d \tau .
$$

Ce dernier est solution du système d'équation suivant :

$$
\begin{cases}\Delta \Psi=0 & z \leq 0 \\ \Psi=0 & z=0 \text { et }|x|>d \\ \frac{\partial \Psi}{\partial z}=\vec{U}_{S} \cdot \vec{z}+f(x) & z=0 \text { et }|x| \leq d \\ \Psi \rightarrow 0 & x^{2}+z^{2} \rightarrow 0\end{cases}
$$

Comme nous le voyons, pour déterminer le potentiel des déplacements, il n'est pas nécessaire de connaître l'historique du mouvement du corps impactant, mais uniquement sa position actuelle. L'élévation de la surface libre peut être obtenue à partir du potentiel des déplacements en utilisant la relation suivante :

$$
h(x, t)=\frac{\partial \Psi(x, 0, t)}{\partial z} .
$$
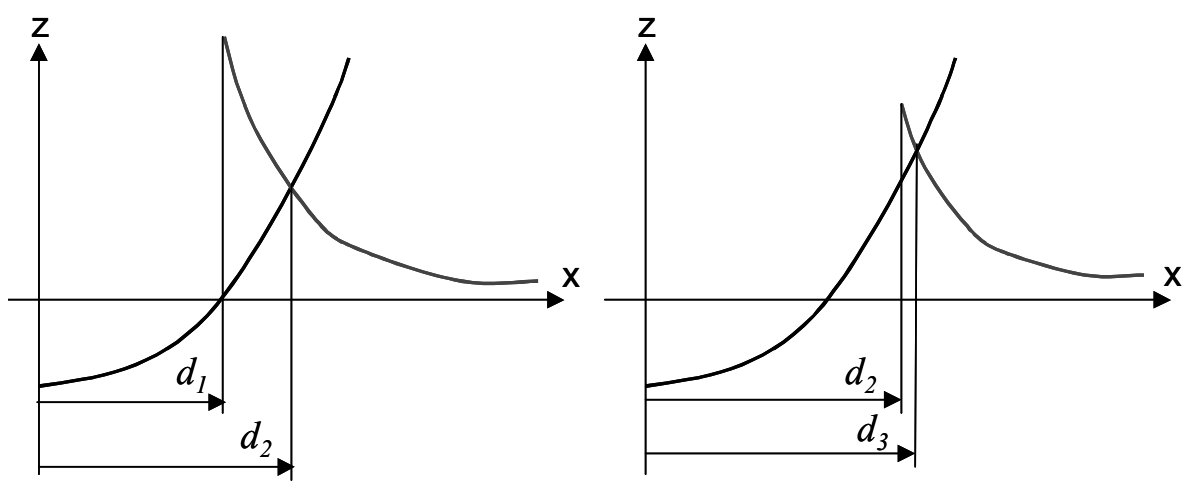

Figure 3. Illustration du fonctionnement de l'algorithme de calcul de la largeur mouillée au cours des deux premières itérations

Dans la pratique, une procédure itérative est utilisée pour calculer la largeur mouillée. Tout d'abord, une première estimation $d_{l}$ de la largeur mouillée est obtenue à partir du point d'intersection entre la surface initiale du fluide et celle du solide à l'instant considéré. Ensuite, on calcule le potentiel des déplacements [12], puis l'élévation de la surface libre [13], en utilisant cette estimation de la largeur mouillée. Comme $d_{l}$ est plus faible que la «vraie » valeur de la largeur mouillée, il existe une interpénétration entre la surface libre (calculée avec $d_{l}$ ) et la surface du solide. Une nouvelle estimation $d_{2}$ de la largeur mouillée est déterminée en prenant le point d'intersection entre ces deux surfaces (figure 3 ). Un nouveau calcul du 
potentiel des déplacements et de l'élévation de la surface libre est réalisé en prenant $d=d_{2}$. Si l'on observe encore une interpénétration entre le solide et la surface libre, une nouvelle estimation de $d$ est réalisée en prenant le point d'intersection. On continue à itérer de la sorte jusqu'à ce que l'interpénétration entre les deux surfaces soit inférieure à une certaine tolérance.

\subsubsection{Calcul de la pression hydrodynamique}

Dans le cadre de la théorie de Wagner originelle, la pression agissant sur le solide impactant est calculée à l'aide de l'équation de Bernoulli linéarisée,

$$
p_{e}=-\rho_{l} \frac{\partial \Phi}{\partial t}
$$

qui est utilisée pour établir la condition de surface libre [9-b]. Cependant, ce champ de pression n'est pas directement exploitable pour calculer l'effort hydrodynamique que subit le corps impactant. En effet, le potentiel des vitesses est singulier à proximité du point d'intersection entre surfaces libre et mouillée et la pression en ce point tend vers l'infini, (Faltinsen, 2005). Cela est lié au fait que dans le cadre de la théorie de Wagner, du fait de la linéarisation des conditions aux limites, l'écoulement au bord de la surface libre et en particulier le jet qui se forme à cet endroit (figure 4) ne sont pas correctement décrits. Ce point avait été mentionné par Wagner (1931) lui-même. Ce dernier suggéra également pour analyser le champ de pression dans cette zone d'utiliser une solution analytique correspondant à la formation d'un jet de fluide parfait sur une plaque rigide. Cette solution est généralement appelée solution intérieure et la solution provenant de la résolution du problème de Wagner [9,14] solution extérieure. Néanmoins, Wagner n'avait pas indiqué comment relier la solution intérieure et la solution extérieure afin obtenir un champ de pression continu, utilisable sur toute la surface du solide. Ce problème fut résolu pour la première fois par Cointe et Arnaud (1987) en utilisant la méthode des développements asymptotiques raccordés. Cette méthode a également été utilisée par Zhao et Faltinsen (1993) qui ont obtenu le champ de pression agissant sur le solide impactant $p_{c}$ sous une forme dite « composite » :

$$
p_{c}=p_{e}+p_{i}-p_{\text {rac }},
$$

où $p_{e}$ est appelée pression extérieure, qui provient de la résolution du problème de Wagner [9,14], $p_{i}$ est la pression intérieure (calculée avec le modèle analytique décrivant l'écoulement à la racine du jet) et $p_{\text {rac }}$ est une pression de raccord qui permet que le champ de pression final soit continu. Le champ de pression utilisé dans Impact++ a aussi une forme «composite» et utilise également la solution intérieure de Wagner pour déterminer la pression à proximité du bord de la surface mouillée. Les expressions de $p_{i}$ et $p_{\text {rac }}$ qui sont utilisées étant un peu longues, ne sont pas reproduites dans cet article. Le lecteur désirant avoir plus d'informations à ce sujet peut se reporter à (Constantinescu, 2006). 


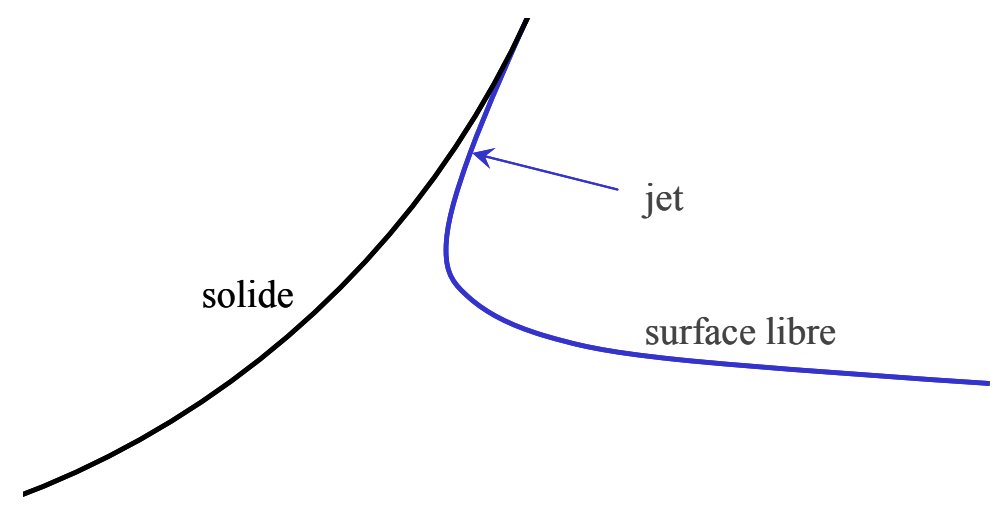

Figure 4. Illustration de l'écoulement réel au bord de la surface libre. Un jet se forme le long de la surface du solide

\subsubsection{Mise en æuvre numérique}

Le calcul du champ de pression «extérieure » [14] nécessite de connaître la dérivée par rapport au temps du potentiel des vitesses. Cette dernière est estimée à l'aide d'une formule de différence finie :

$$
p_{e}(t)=-\rho_{l} \frac{\Phi(t+\delta t)-\Phi(t)}{\delta t} .
$$

Le calcul de la pression nécessite donc de déterminer le potentiel des vitesses à deux instants différents. Les calculs de potentiel des vitesses se font à l'aide d'une résolution par éléments finis du système d'équation [9]. Pour cela, il est tout d'abord nécessaire de calculer à chacun des deux instants la largeur mouillée à l'aide de la procédure itérative présentée dans la section 2.1.2. Cette dernière requière le calcul par éléments finis du potentiel des déplacements à chaque itération. L'ensemble des opérations nécessaires pour le calcul de la pression «extérieure » est illustré par la figure 5 .

D’un point de vue informatique, le développement d'Impact++ n'a pas nécessité la programmation d'un modèle éléments finis spécifique pour les calculs des potentiels des vitesses $\Phi$ et des déplacements $\Psi$, mais repose sur une analogie fluidethermique. En effet, les systèmes d'équations [9] et [12] sont similaires à ceux qui régissent les problèmes de conduction thermique en régime permanent. Cela nous permet, pour le calcul de $\Phi$ et de $\Psi$, d'utiliser le module de thermique d'un code existant, en l'occurrence le code ABAQUS. L'ensemble de la procédure de calcul a été codé à l'aide d'un programme écrit en fortran et l'interfaçage avec ABAQUS est réalisé à l'aide de scripts python. Le domaine qui est utilisé pour les calculs éléments finis représente un quart de cercle. Sa taille évolue au cours du calcul ; elle est égale à 8 fois la largeur mouillée. Ce domaine est maillé à l'aide d'éléments de transfert thermique triangulaires à interpolation linéaire (désignés dans la documentation 
ABAQUS par DC2D3 pour les problèmes plans et DCAX3 pour les problèmes axisymétriques). Le maillage utilisé comporte environ 11000 éléments.

REMARQUe. - Pour des raisons de précision, le pas de temps $\delta t$ qui est utilisé pour le calcul de la pression [16] est différent du pas de temps $\Delta t$ de la simulation (qui représente l'intervalle de temps entre deux instants où l'on calcule la pression hydrodynamique). Dans le cas de simulations d'impacts de solides rigides, le pas de temps $\Delta t$ peut être choisi librement, car comme nous l'avons déjà précisé, dans le cadre de la théorie de Wagner, aussi bien la largeur mouillée que le champ de potentiel des vitesses peuvent être déterminés en connaissant uniquement la position actuelle du corps impactant. Bien sûr, il n'en va pas de même lorsque le modèle d'impact hydrodynamique que nous avons présenté est couplé à un code de calcul de structures pour traiter des problèmes d'impact hydroélastique. Dans ce cas, la précision des résultats, ainsi que la stabilité des simulations, nécessite d'utiliser un pas de temps $\Delta t$ suffisamment petit.

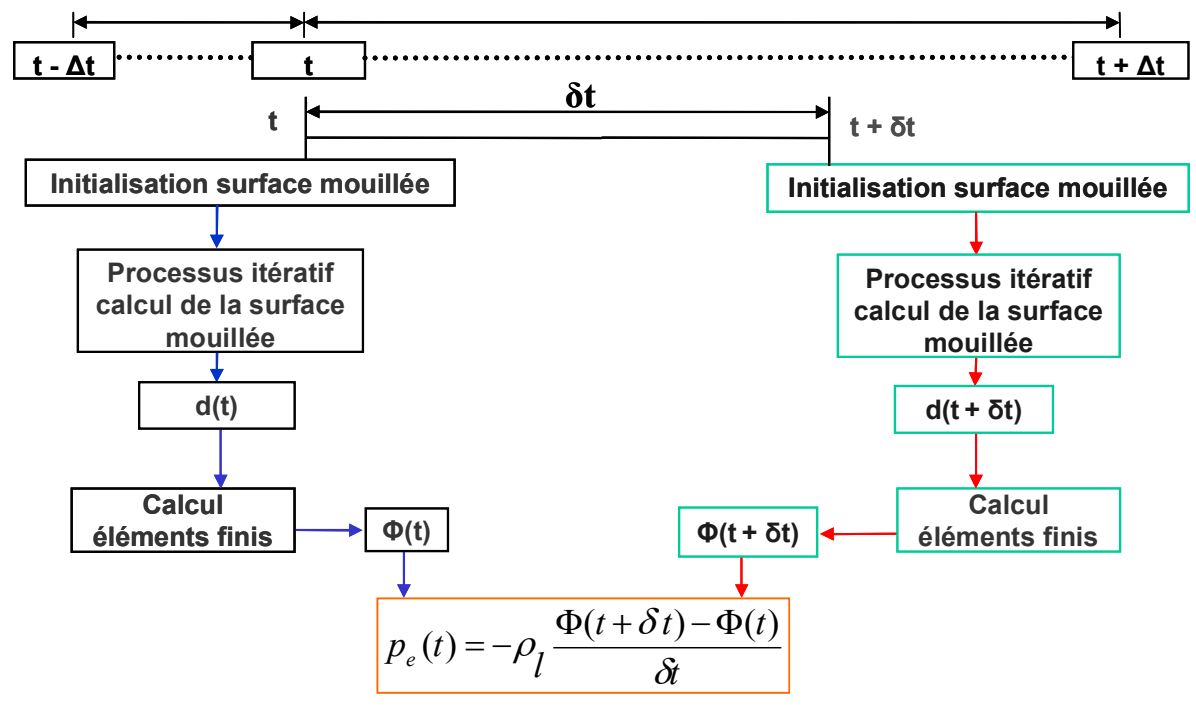

Figure 5. Organigramme d'un calcul avec Impact ++

\subsection{Modèle éléments finis basé un formalisme arbitraire Lagrange-Euler (ALE)}

Ces simulations ont été réalisées à l'aide du code ABAQUS/Explicit. Cette approche impose de prendre en compte la compressibilité du fluide. Nous avons pour cela choisi d'utiliser une équation d'état de Mie-Gruneisen :

$$
p=\frac{\rho_{0} c_{0}^{2} \eta}{(1-s \eta)^{2}}\left(1-\frac{\Gamma_{0} \eta}{2}\right)+\Gamma_{0} \rho_{0} E_{m}, \quad \text { avec } \quad \eta=1-\frac{\rho_{0}}{\rho_{l}}
$$


où $p$ est la pression, $\rho_{l}$ la masse volumique et $E_{m}$ l'énergie interne par unité de masse. Les paramètres de cette équation sont $\rho_{0}$ la masse volumique initiale, $c_{0}$ la célérité initiale des ondes acoustiques. Les paramètres $s$ et $\Gamma_{0}$ ont été pris égaux à zéro. En effet, ils n'ont de l'influence que pour les très forts niveaux de pression (rappelons que l'équation de Mie-Gruneisen est généralement utilisée pour modéliser la propagation des ondes de chocs dans les solides). Bien que ce ne soit pas absolument nécessaire, la viscosité du fluide est également prise en compte :

$$
\underline{\underline{\hat{\sigma}}}=2 \mu \underline{\underline{\hat{D}}}
$$

$\underline{\underline{\hat{\sigma}}}$ et $\underline{\underline{D}}$ sont respectivement le déviateur du tenseur des contraintes de Cauchy et celui du tenseur vitesse de déformation, $\mu$ est la viscosité dynamique du fluide.

Le principe des formulations ALE est de dissocier le mouvement de la matière et celui du maillage. Ceci permet de limiter les problèmes de distorsion du maillage qui peuvent intervenir lors des simulations purement lagrangiennes. Le code ABAQUS utilise une formulation ALE particulière appelée méthode «split». Ainsi, les simulations sont divisées en une succession de phases de calcul lagrangiennes durant lesquelles le maillage se déforme en suivant le mouvement de la matière et de phases d'adaptation de maillage. Lors de ces dernières le mouvement de la matière est figé et les opérations suivantes sont effectuées : tout d'abord, les nœuds du maillage sont repositionnés afin de réduire la distorsion des éléments. Ensuite, la solution (variables aux nœuds et points d'intégration des éléments) est transférée de l'ancien vers le nouveau maillage. La méthode d'advection qui est utilisée pour cela par ABAQUS est basée sur les travaux de Van Leer (1977). On reprend ensuite une nouvelle phase de calcul lagrangienne. Durant ces dernières, l'intégration temporelle des équations du mouvement est réalisée à l'aide du schéma des différences centrées.

Le maillage du domaine fluide comporte deux régions. A proximité de la zone d'impact, nous utilisons un maillage ALE constitué d'éléments quadrangulaires à interpolation bilinéaire et intégration réduite (désignés dans la documentation ABAQUS par CPE4R pour les problèmes plans et CAX4R pour les problèmes axisymétriques). Dans le reste du domaine, la déformation du fluide est très faible. Par conséquent, pour cette région, un maillage Lagrangien constitué d'éléments triangulaires, dont la taille augmente progressivement avec la distance de la zone d'impact, est utilisé (CPE3 et CAX3). Les maillages utilisés comportent entre 70000 et 170000 éléments.

Le solide impactant est modélisé à l'aide d'une surface rigide. L'interaction entre le solide et le fluide est prise en compte en définissant une condition de contact. Concernant la gestion du contact, une méthode de type prédicteur-correcteur (appelée « kinematic predictor-corrector contact algorithm » dans la documentation ABAQUS) a été utilisée. De manière très schématique, le fonctionnement de cet algorithme peut être décrit de la façon suivante : à chaque pas de temps, le code va tout d'abord intégrer les équations du mouvement sans prendre en compte le contact (étape de prédiction). Ensuite, au niveau de chaque nœud du maillage 
(correspondant au fluide dans notre cas) qui a franchi la surface maître lors de l'étape de prédiction, le code va déterminer l'effort de contact à appliquer à ce nœud afin que la loi de contact soit vérifiée de manière exacte à la fin du pas de temps et refaire l'intégration du mouvement du nœud en question en tenant compte de cet effort de contact (étape de correction) ${ }^{1}$.

REMARQUE. - Choix de la taille du domaine fluide.

Notre objectif est de calculer les efforts hydrodynamiques s'exerçant sur une structure pénétrant un domaine fluide supposé infini. Il est donc important d'utiliser dans les simulations un domaine fluide dont la taille est suffisamment importante pour éviter les effets de confinement. La compressibilité étant prise en compte, on peut choisir la taille du domaine en remarquant que la structure impactante ne verra pas l'effet des conditions aux limites si les ondes se réfléchissant sur les bords du domaine n'ont pas le temps de revenir sur celle-ci, c'est-à-dire si la condition suivante est respectée :

$$
L \geq c_{0} T / 2
$$

où $L$ est la distance minimale entre les bords du domaine et la structure impactante et $T$ est la durée physique que l'on désire simuler.

\subsection{Modèle volumes finis basé sur un formalisme eulérien multiphasique}

\subsubsection{Présentation générale}

Le code de CFD commercial Fluent a été utilisé pour réaliser ces simulations. Nous considérons une phase liquide (eau) et une phase gazeuse (air), qui sont supposées immiscibles. L'interaction entre ces deux phases est prise en compte à l'aide du modèle de volume fractionnel de fluide (encore appelé méthode «Volume of Fluid» ou VOF), (Hirt et Nichols, 1981). Avec ce modèle, des variables supplémentaires sont rajoutées au niveau de chaque cellule du maillage : la fraction volumique de chaque fluide en présence. Ainsi, les mailles peuvent être remplies d'air ou d'eau, mais aussi d'un mélange des deux. Avec la méthode VOF, un seul jeu d'équations pour la conservation de la masse et de la quantité de mouvement est résolu pour l'ensemble des fluides. Dans nos simulations, le comportement des fluides est gouverné par les équations de Navier-Stokes. Lorsqu'une cellule est partiellement remplie, des propriétés mécaniques (masse volumique et viscosité) équivalentes sont définies à l'aide de lois des mélanges :

$$
\rho=\rho_{l} \alpha_{l}+\rho_{g}\left(1-\alpha_{l}\right) \quad \text { et } \quad \mu=\mu_{l} \alpha_{l}+\mu_{g}\left(1-\alpha_{l}\right),
$$

1. Nous noterons que le fonctionnement de cet algorithme repose sur le fait que les codes de dynamique explicite tels que ABAQUS utilisent des matrices de masse condensées (diagonalisées), voir par exemple (Belytschko et al., 2000). 
où $\rho_{l}, \alpha_{l}$ et $\mu_{l}$ sont respectivement la masse volumique, la fraction volumique et la viscosité de l'eau; $\rho_{g}$ et $\mu_{g}$ correspondent à la phase gazeuse. L'évolution spatiotemporelle de la fraction volumique d'eau $\alpha_{l}$ est gouvernée par l'équation de transport suivante :

$$
\frac{\partial\left(\rho_{l} \alpha_{l}\right)}{\partial t}+\operatorname{div}\left(\rho_{l} \alpha_{l} \vec{v}\right)=0 .
$$

La compressibilité de l'eau a été considérée pour la plupart des simulations. L'intérêt de la prise en compte de la compressibilité de la phase liquide est discuté dans le prochain paragraphe. Pour cela, il faut créer une UDF (User Defined Function) dans laquelle on doit calculer la masse volumique $\rho_{l}$ en fonction de la pression $p$; l'équation d'état suivante à été programmée :

$$
\rho_{l}=\rho_{0}\left(1-\frac{p-p_{0}}{K}\right)^{-1}
$$

où $p_{0}$ est une pression de référence et $K$ est le module de compressibilité du fluide (notons que $K=\rho_{0} c_{0}^{2}$ ). Il est aussi nécessaire de définir dans l'UDF, une équation permettant de calculer la célérité $c_{l}$ des ondes dans le fluide :

$$
c_{l}=\left(1-\frac{p-p_{0}}{K}\right) \sqrt{\frac{K}{\rho_{0}}}
$$

Il aurait été possible de prendre en compte la compressibilité de l'air à l'aide de la loi des gaz parfaits. Néanmoins, cela conduit à une augmentation significative des temps de calcul, car d'une part, Fluent résout dans ce cas un jeu d'équations supplémentaire correspondant à la conservation de l'énergie, et, d'autre part, la prise en compte de la compressibilité de l'air peut conduire à des difficultés de convergence. Pour les simulations présentées dans cet article, l'air est modélisé comme incompressible. Mais, même sous cette hypothèse, comme la densité de l'air est faible devant celle de l'eau, la présence de l'air a un effet négligeable sur les résultats.

La discrétisation spatiale des équations de conservation de la masse et de la quantité de mouvement repose sur l'utilisation d'un schéma volumes finis décentré d'ordre 2 . La discrétisation de l'équation de transport pour la fraction volumique [21] nécessite l'utilisation de schémas particuliers pour éviter une diffusion trop importante de l'interface entre les phases. Nous avons utilisé une méthode de type « geo-reconstruct» (Youngs, 1982). Dans cette dernière, afin de calculer avec plus de précision les flux au niveau des faces entre cellules, la position de la surface libre est estimée à partir de la valeur de la fraction volumique et de son gradient au centre des cellules, en se basant sur l'hypothèse que cette surface est linéaire au sein d'une cellule.

Pour prendre en compte le déplacement du solide impactant, une possibilité serait d'utiliser les outils de remaillage automatique disponibles dans Fluent. Toutefois, cette approche peut conduire à une source d'imprécision supplémentaire (Ferziger et Perić, 
2002). Par conséquent, pour éviter de devoir remailler, les simulations considèrent l'impact sur un solide fixe d'une colonne d'eau, dont la vitesse initiale correspond à la vitesse d'impact retenue (figure 6). Lorsque cela est possible, le domaine de calcul est maillé à l'aide de quadrangles de manière à obtenir un maillage structuré par blocs. Pour certaines géométries, il a été nécessaire d'utiliser aussi des triangles, mais uniquement dans des zones éloignées du corps impactant. Les maillages utilisés pour ces simulations comportent entre 60000 et 200000 cellules.

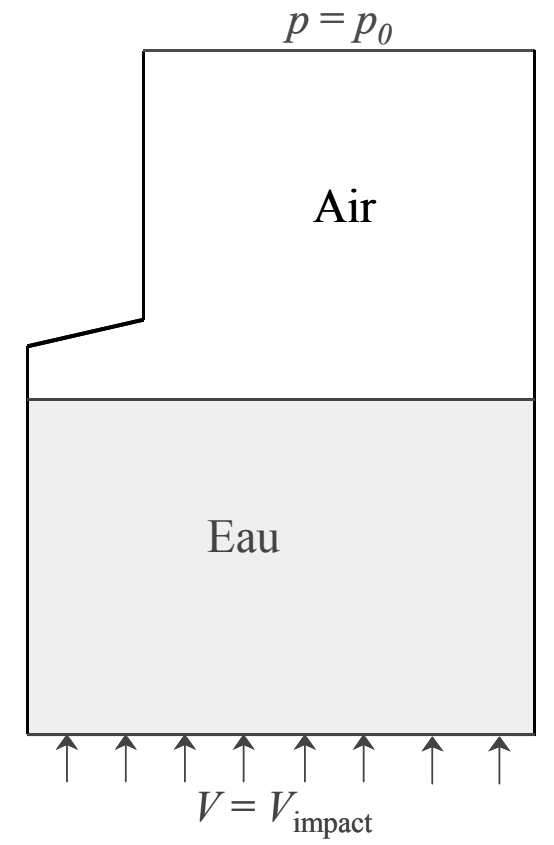

Figure 6. Exemple de configuration utilisée lors des simulations réalisées avec Fluent

\subsubsection{Faut-il prendre en compte la compressibilité du fluide?}

Comme nous l'avons déjà indiqué, dans la plupart des travaux portant sur l'impact hydrodynamique, la compressibilité du fluide est généralement négligée. Néanmoins, comme nous allons le voir, pour les simulations Fluent, il est préférable de la prendre en compte.

La figure 7 montre l'évolution temporelle de la force hydrodynamique s'exerçant sur un dièdre. Des résultats obtenus avec un fluide compressible et incompressible sont présentés. De plus, plusieurs tailles de domaine sont considérées. Nous rappelons que nous voulons minimiser les effets de confinement liés à la taille finie du domaine de calcul. Comme cela a été indiqué dans la section 2.2, dans le cas compressible, on peut déterminer, en considérant la vitesse des ondes, une taille du domaine permettant 
d'éliminer l'effet des conditions aux limites sur les pressions qui s'exercent sur le dièdre [19]. Ceci n'est bien sûr pas possible lorsque le fluide est incompressible, l'influence de la taille du domaine se faisant sentir tout au long de la simulation. Nous avons observé que, dans le cas incompressible, il est difficile de s'affranchir des effets de confinement, même en prenant des tailles de domaine relativement grandes $(1,5 \mathrm{~m})$ par rapport à la demi-largeur du dièdre $(0,128 \mathrm{~m})$. Pour illustrer ces propos, observons les courbes de la figure 6. On constate qu'au début de l'impact, les courbes sont confondues et l'augmentation de l'effort est linéaire par rapport au temps. Remarquons, que pour un dièdre impactant à vitesse constante un fluide infini, la linéarité de la force hydrodynamique par rapport au temps peut être démontrée théoriquement par une analyse dimensionnelle. Dans le cas incompressible, on voit que, pour des temps plus importants, la courbe s'éloigne de cette tendance théorique et que cet effet est d'autant plus marqué que la taille du domaine fluide est réduite. Ceci montre très clairement l'effet de confinement. On constate également que les courbes obtenues avec un fluide incompressible présentent un bruit numérique assez important. Ce phénomène a déjà été observé lors de simulations d'écoulements biphasiques de fluides incompressibles et est dû principalement au manque de résolution concernant la position de l'interface (Ganesan et al., 2007). La prise en compte de la compressibilité permet d'éviter ce problème.

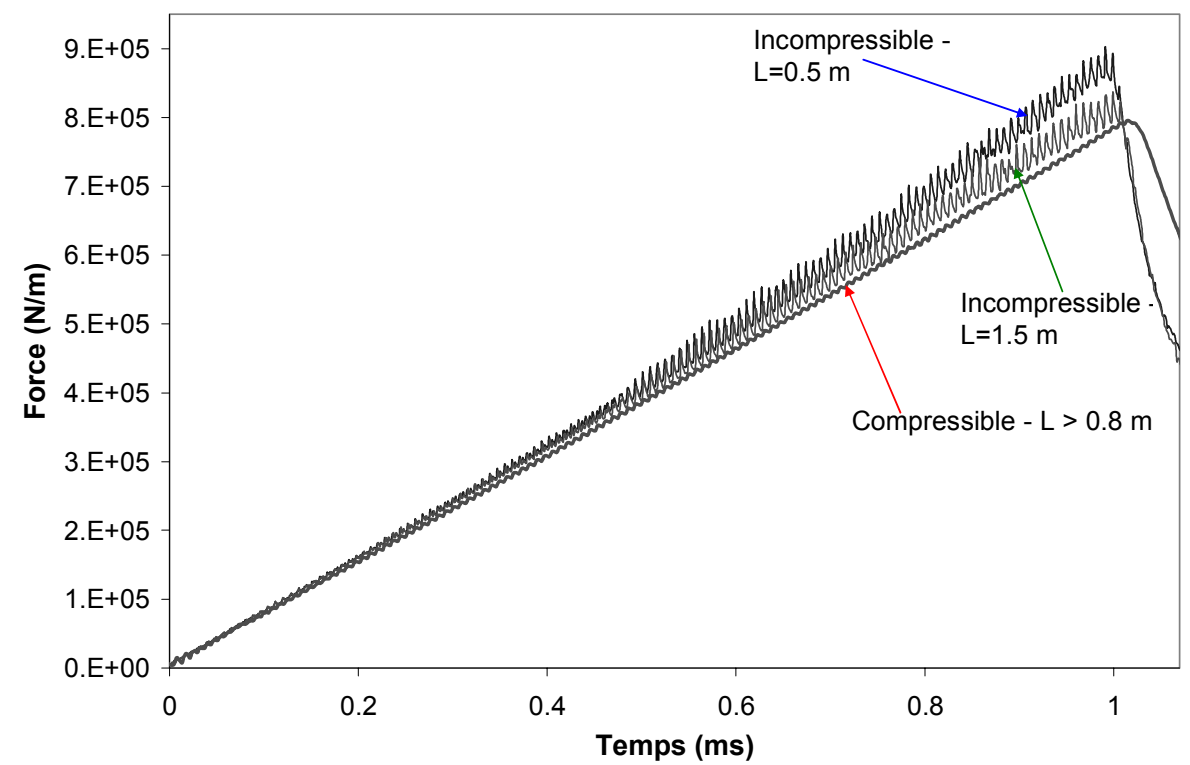

Figure 7. Effet de la compressibilité et de la taille du domaine fluide lors de la simulation de l'impact d'un dièdre avec Fluent. La composante verticale de la force hydrodynamique exercée sur le dièdre est représentée. Vitesse d'impact : $V=20$ $\mathrm{m} / \mathrm{s}$, angle du dièdre : $\beta=14^{\circ}$ 
$\mathrm{Au}$ final, nous pouvons conclure qu'il est préférable de prendre en compte la compressibilité, non pas parce qu'elle a un effet important dans le phénomène étudié, mais parce qu'elle facilite la mise en oeuvre des calculs en permettant d'utiliser des maillages moins volumineux et en évitant le phénomène de bruit numérique. Notons que la prise en compte de la compressibilité sera également un avantage pour la simulation et l'analyse d'essais réalisés en bassin dont la taille est forcément limitée.

\section{Résultats et discussions}

Dans cette section, nous allons considérer, à l'aide des trois modèles qui viennent d'être présentés, l'impact hydrodynamique de solides de différentes formes. Nous avons également inclus certains résultats de la littérature, ainsi que des résultats expérimentaux. Pour décrire le comportement de l'eau, les paramètres suivants ont été utilisés : $\rho_{0}=1000 \mathrm{~kg} / \mathrm{m} 3, c_{0}=1424 \mathrm{~m} / \mathrm{s}$ et $\mu=0.001$ Pa.s (pour certains modèles, il n'est pas nécessaire de définir l'ensemble de ces trois paramètres).

\subsection{Dièdres}

Considérons tout d'abord le cas de dièdres impactant une surface d'eau à vitesse constante. Les résultats présentés sont l'effort hydrodynamique total et l'évolution temporelle de la pression au niveau de deux points de la surface des dièdres, dont la position est illustrée par la figure 8 .

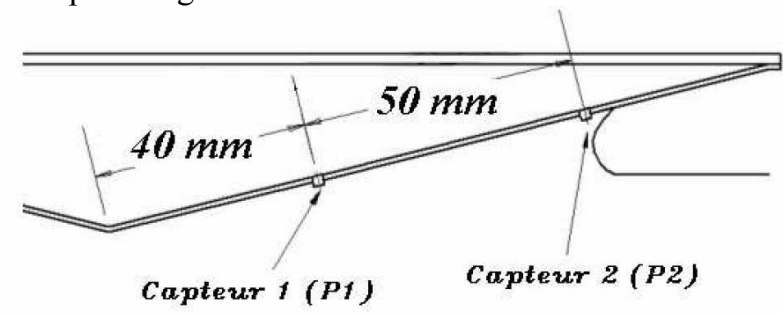

Figure 8. Illustration de la géométrie de dièdres et position des points où la pression est mesurée

La figure 9 montre les résultats obtenus avec un dièdre dont l'angle de pénétration (angle entre la surface du dièdre et la surface initiale de l'eau) est égal à $14^{\circ}$. En plus des résultats obtenus à l'aide des trois modèles numériques, nous avons également inclus ceux obtenus à l'aide du modèle analytique de Zhao et Faltinsen (1993), qui est également basé sur la théorie de Wagner. En ce qui concerne l'effort total s'exerçant sur le dièdre (figure 9a), nous observons que les résultats obtenus avec Fluent sont extrêmement proches de ceux obtenus avec ABAQUS/Explicit. Les résultats du code 
Impact++ sont, quant à eux, très proches de ceux obtenus avec le modèle analytique de Zhao et Faltinsen. Entre les résultats Fluent et ABAQUS/Explicit d'une part, et ceux d'Impact++ et de Zhao et Faltinsen d'autre part, on observe un écart d'environ $12 \%$. Comme nous le verrons par la suite, cette différence semble être due aux hypothèses simplificatrices sur lesquelles repose la théorie de Wagner. Considérons maintenant les courbes montrant l'évolution temporelle de la pression au niveau de deux points de la surface du dièdre (figure 9b). Initialement, ces deux points ne sont pas immergés et la pression est nulle. Lorsque l'eau arrive au niveau des capteurs, la pression augmente très fortement et atteint très rapidement un pic. La pression redescend ensuite mais conserve un niveau assez important. Concernant les pics de pression, les quatre modèles prévoient des valeurs assez proches de la pression maximale. Par contre, un léger décalage existe en ce qui concerne l'instant de ces pics. Ceux prévus par Impact++ et le modèle Zhao et Faltinsen surviennent un peu plus tôt que ceux prévus par Fluent et ABAQUS/Explicit. Au niveau du point P2, ce décalage représente $22 \mu \mathrm{s}$, soit un écart relatif de $3 \%$.

Une synthèse des résultats obtenus dans le cas des dièdres est présentée par la figure 10. Comme nous l'avons indiqué précédemment, pour un dièdre impactant une surface d'eau à vitesse constante l'effort hydrodynamique augmente linéairement au cours du temps, il est donc commode de représenter les résultats à l'aide du nombre sans dimension suivant ${ }^{2}$ :

$$
f_{D}(\beta)=\frac{F(t) \tan (\beta)^{2}}{\rho_{l} V^{3} t}
$$

où $F$ est l'effort hydrodynamique (par unité de longueur), $V$ la vitesse d'entrée dans l'eau, $\rho_{l}$ la masse volumique du fluide et $\beta$ l'angle du dièdre. En plus des résultats des 3 modèles numériques et de ceux du modèle de Zhao et Faltinsen, nous avons également inclus sur la figure 10 des résultats théoriques qui ont été obtenus à l'aide la méthode des similitudes par Zhao et Faltinsen (1993), ainsi que les résultats obtenus par Iafrati et al. (2000). La méthode des similitudes repose sur l'hypothèse d'un fluide parfait incompressible. Par contre, aucune autre hypothèse (en particulier concernant la forme de la surface du fluide) n'est faite. Si bien que ces résultats sont considérés par beaucoup d'auteurs comme des résultats de référence dans le cas de l'impact de dièdres à vitesse constante. Iafrati et al. (2000) ont utilisé quant à eux un modèle éléments de frontière basé sur la théorie du potentiel. Ce modèle est également totalement non linéaire ; dans ces simulations, la géométrie de la surface libre du fluide est mise à jour tout au long du calcul à partir de la connaissance du champ de vitesse et les conditions aux limites sont imposées sur cette surface. La

2. Notons qu'en toute rigueur, les résultats obtenus avec les modèles prenant en compte la compressibilité du fluide, ne devraient pas être représentés sur ce graphique. Néanmoins, les résultats des simulations montrent que, pour les cas présentés dans cet article, l'effet de la compressibilité est très faible et donc la valeur de $f(\beta)$ ne dépend pratiquement pas de la vitesse d'impact. 
figure 10 nous montre que les résultats obtenus avec Fluent et ABAQUS/Explicit, ainsi que ceux obtenus par Iafrati et al. (2000), sont tous très proches des résultats de la méthode des similitudes. Les efforts prévus par impact++ et le modèle de Zhao et Faltinsen sont, quant à eux, plus élevés. En outre, l'écart croît lorsque l'angle de pénétration augmente. Il est raisonnable de penser que cette différence est due aux hypothèses simplificatrices de la théorie de Wagner, en particulier celle qui consiste à négliger la déformation de la surface du fluide. On comprend aisément que cette hypothèse est de moins en moins valide lorsque l'angle d'incidence du dièdre augmente. Concernant les résultats Impact++ et du modèle de Zhao et Faltinsen, nous voyons qu'ils sont très proches pour des angles inférieurs à $15^{\circ}$, une différence apparaissant pour des valeurs plus importantes de l'angle. Cette différence s'explique par le fait que le champ de pression «composite» [15] utilisé dans Impact++ n'est pas exactement le même que dans le modèle de Zhao et Faltinsen.

Des simulations d'impact d'un dièdre ayant un angle de $10^{\circ}$ ont été réalisées par Aquelet et al. (2006). Ces simulations sont basées sur la méthode VOF et sur un algorithme de couplage fluide-structure utilisant une méthode de pénalisation. Ces auteurs trouvent une valeur de $f_{D}\left(10^{\circ}\right)$ égale à environ 7 . Si on la compare aux résultats de la figure 10, cette valeur se situe entre les résultats des modèles basés sur la théorie de Wagner et les modèles complètement non linéaires. Ceci est un peu surprenant car le modèle utilisé par Aquelet et al., (2006) fait partie de la seconde catégorie.

REMARQUE. - Temps de calcul. Il est difficile de faire une comparaison définitive des différents codes en termes de temps de calcul, car leur efficacité dépend beaucoup de la configuration considérée (vitesse et angle de pénétration). Par exemple, avec ABAQUS/Explicit, la durée du calcul dépend fortement de la durée de temps que l'on désire simuler. En effet, la taille des pas de temps qu'il utilise est fixée par la limite de stabilité du schéma des différences centrées qui est estimée par ABAQUS par la relation suivante :

$$
\Delta t_{\text {crit }} \leq \min \left(L_{e} / c\right),
$$

où $\Delta t_{c r i t}$ est la taille maximale de pas de temps utilisable, $L_{e}$ est la longueur caractéristique des éléments et $c$ est la vitesse des ondes acoustiques. Par conséquent, lorsque l'on réduit la vitesse d'impact et donc que l'on augmente la durée de cet impact, le nombre de pas de temps nécessaire et donc le temps de calcul augmentent fortement. Ainsi, avec ABAQUS/Explicit, les cas d'impact les plus violents (faibles angles de pénétration et fortes vitesses) sont ceux qui nécessitent les temps de calcul les plus faibles. Avec Fluent, pour les simulations impliquant la méthode VOF, la discrétisation temporelle repose sur l'utilisation d'un schéma de type Euler implicite qui n'a pas de telle limite de stabilité. Néanmoins, dans la pratique, l'utilisation de pas de temps trop grands dégrade la qualité des résultats et cause des difficultés de convergence. Nous avons observé que pour simuler les impacts à vitesse élevée et faible angle de pénétration, il est nécessaire d'utiliser des pas de temps très faibles, ce qui engendre des temps de calcul importants. Nous allons simplement donner un ordre de grandeur des temps de calcul moyens. Sur un 
ordinateur de bureau (processeur Pentium $43 \mathrm{GHz}$ avec 2 Go de mémoire vive), une simulation avec Impact++ demande environ trois heures. Une douzaine d'heures est nécessaire avec ABAQUS/Explicit et environ trois jours avec Fluent.

(a)

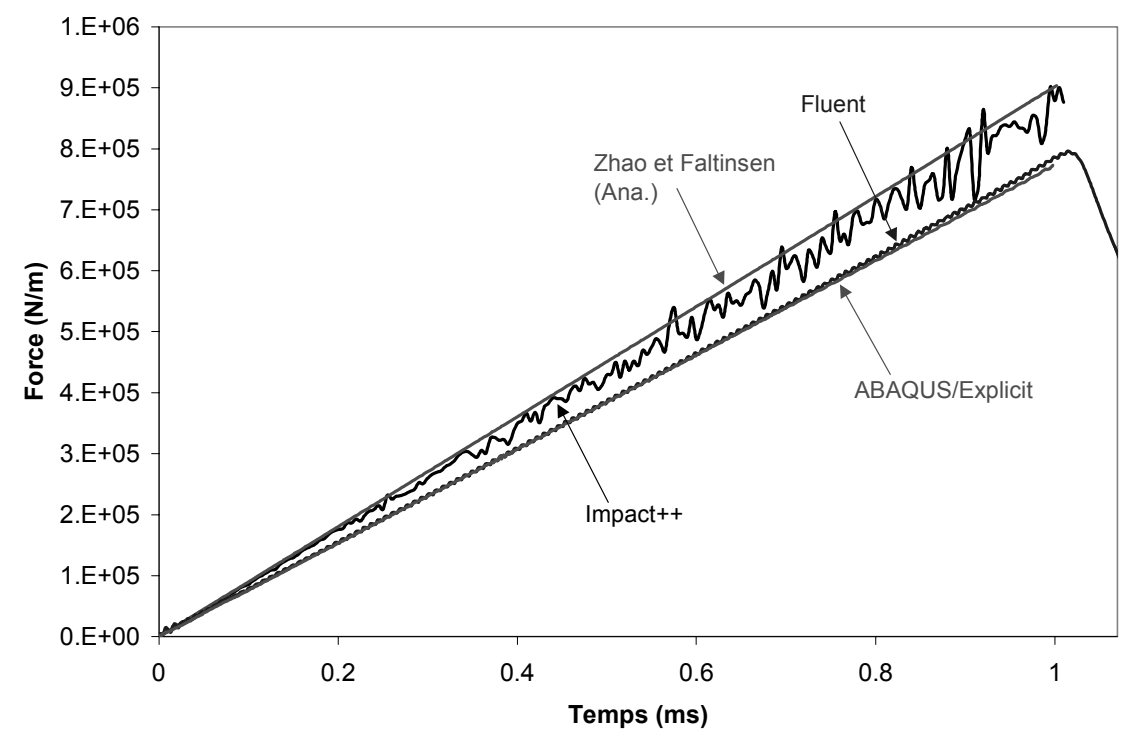

(b)

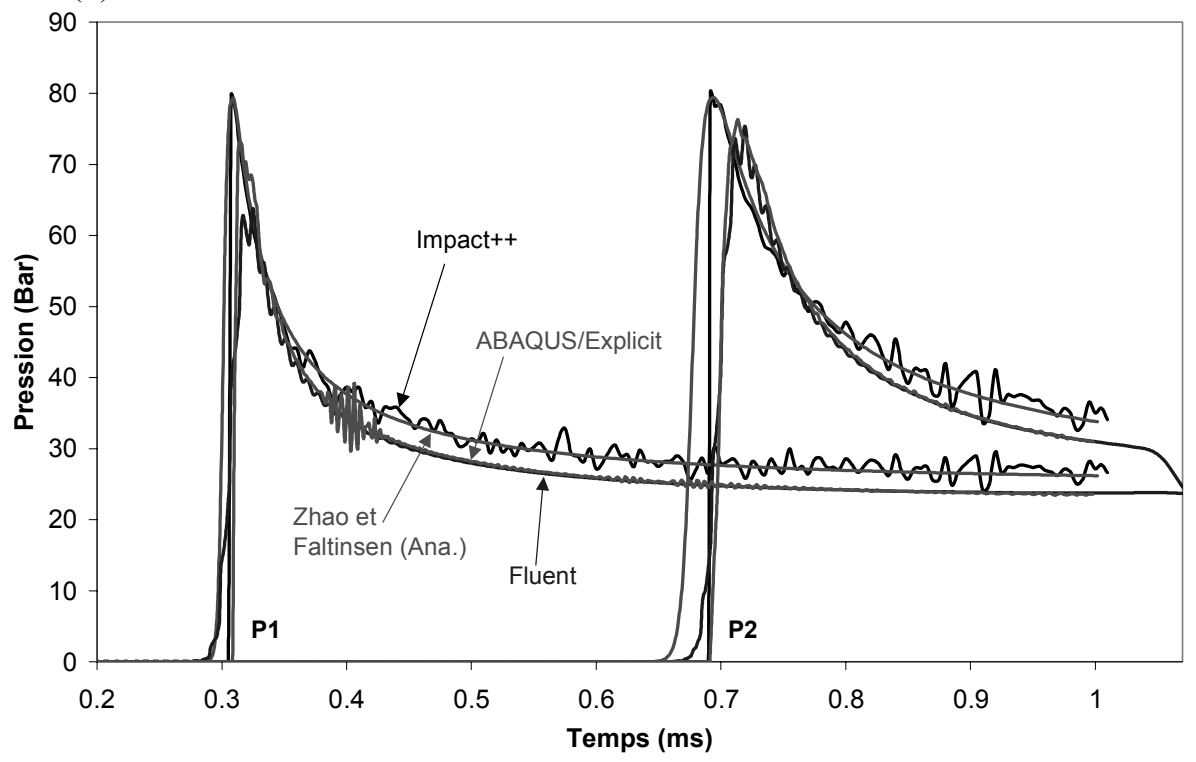

Figure 9. Impact hydrodynamique d'un dièdre rigide avec un angle de pénétration de $14^{\circ}$. La vitesse d'impact est égale à $20 \mathrm{~m} / \mathrm{s}$. (a) Effort hydrodynamique total, (b) pression au niveau des capteurs de pression (figure 8) 


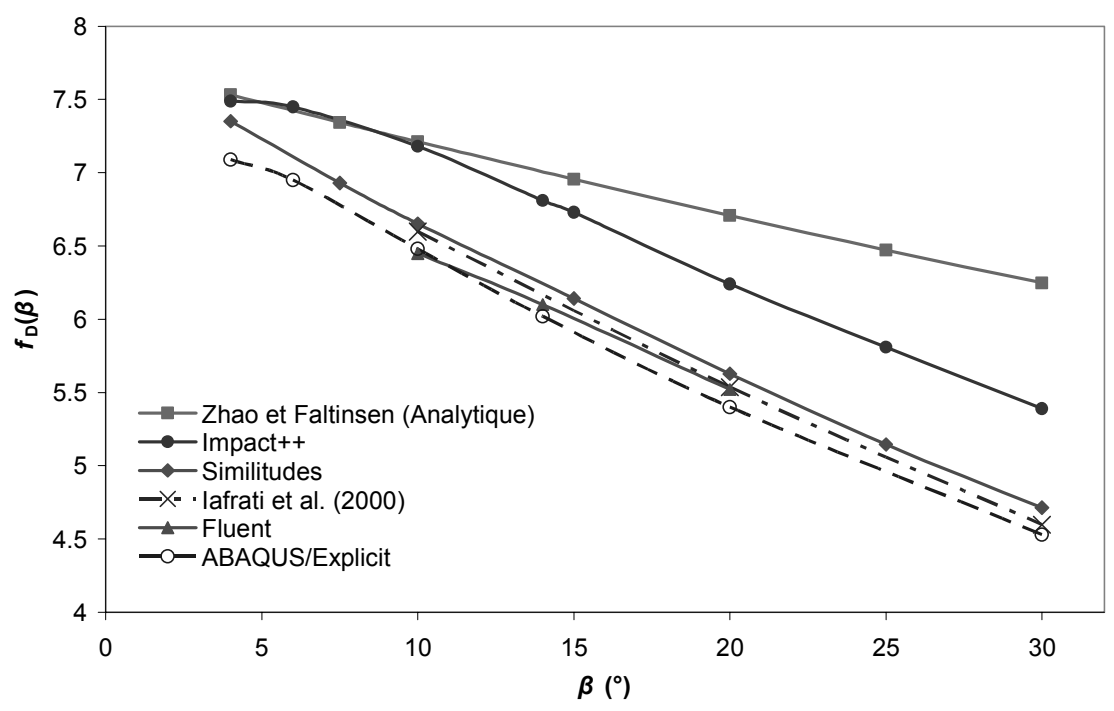

Figure 10. Impact de dièdres à vitesse constante: effort hydrodynamique adimensionnalisé [24] en fonction de l'angle de pénétration

\subsection{Cônes}

La figure 11 montre des résultats pour l'impact d'un cône rigide ayant un angle de pénétration de $6^{\circ}$. Cette valeur d'angle est faible et correspond bien au domaine d'applicabilité de la théorie de Wagner. Ainsi, la force hydrodynamique prédite par Impact++ est proche des résultats obtenus avec Fluent et ABAQUS/Explicit (la différence est d'environ $6 \%$ ). On remarque également que, concernant l'instant des pics de pression aux niveaux des capteurs $\mathrm{P} 1$ et $\mathrm{P} 2$, les résultats des trois codes sont très proches. Par contre, nous remarquons qu'Impact++ prévoit que la valeur du pic de pression ne dépend pas de la position du capteur. De leur côté, Fluent et ABAQUS/Explicit prédisent une valeur plus importante de la pression maximale au niveau du capteur $\mathrm{P} 2$ comparativement à $\mathrm{P} 1$ (tableau 1). Ce phénomène est en fait un artefact numérique. En effet, des résultats théoriques montrent que lors de l'impact d'un cône à vitesse constante, le champ de pression est autosimilaire (Battistin et Iafrati, 2003 ) et sa valeur maximale ne doit pas changer. Il semble donc que la prédiction des pics de pression avec Fluent et ABAQUS/Explicit soit assez délicate dans le cas de faibles valeurs de l'angle de pénétration. La raison en est que ce pic a une largeur très faible. Cependant, cette largeur augmente au cours du temps (c'est pourquoi la durée du pic de pression est plus importante au niveau du capteur P2 que P1). Pour les simulations Fluent et ABAQUS/Explicit, nous utilisons des maillages dont la taille des mailles est constante dans la zone d'impact. Par conséquent, lorsque la simulation progresse, le pic de pression s'appuie sur un nombre de mailles qui est de plus en plus important. Il est donc de mieux en mieux représenté, ce qui explique que la valeur 
maximale de la pression augmente au cours du temps. De son coté, Impact++ n'est pas affecté par ce problème car la pression au niveau du pic dépend uniquement de la pression « intérieure » (voir équation [15]). Comme nous l'avons indiqué, cette dernière peut être vue comme un terme correctif introduit pour supprimer la singularité qui apparaît dans la théorie de Wagner au bord de la surface mouillée. Ainsi la pression maximale, notée $p_{\max }$, est imposée analytiquement et dépend uniquement de la vitesse d'extension de la surface mouillée $\dot{d}$ au travers de la relation suivante :

$$
p_{\max }=\rho_{l}(\dot{d})^{2} / 2
$$

Des essais d'impact hydrodynamique de cônes ont été réalisés sur la machine hydraulique pour essais d'impact présente à l'ENSIETA (figure 12). Schématiquement, cette dernière est constituée d'un vérin pouvant se mettre en mouvement et s'arrêter rapidement. Sur une partie de la course du vérin, la vitesse peut être maintenue constante (sous réserve que l'effort ne dépasse pas une certaine limite). Pour les essais d'impact sur l'eau, la pièce à tester est fixée sur l'extrémité inférieure du vérin et le bassin est placé sous la machine. Concernant l'instrumentation, des jauges de déformation sont collées en extrémité basse de la tige du vérin, elles permettent de déduire l'effort lors de l'impact. Un capteur de déplacement mesure la position du vérin et un accéléromètre est placé sur le cône. L'instant du premier contact entre le cône et l'eau est détecté par un dispositif électrique qui mesure la brusque variation de la résistance entre le cône et une plaque de cuivre placée dans le bassin survenant lorsque le cône touche l'eau. Trois cônes possédant des angles de pénétration différents $\beta=7$, 15 et $30^{\circ}$ ) ont été testés. Ces cônes ont été dimensionnés afin que leur déformation lors de l'impact soit suffisamment faible pour qu'ils puissent être considérés comme rigides. Le tableau 2 donne, pour les différents cônes, les efforts adimensionnalisés qui ont été mesurés lors des essais et calculés à l'aide des modèles numériques. Dans le cas des cônes, l'effort adimensionnalisé est pris sous la forme suivante :

$$
f_{C}(\beta)=\frac{F(t) \tan (\beta)^{3}}{\rho_{l} V^{4} t^{2}} .
$$

Dans le cas des angles de 7 et $15^{\circ}$, nous observons que les résultats des différents modèles numériques sont très proches les uns des autres et en excellent accord avec les résultats expérimentaux. Pour l'angle de $30^{\circ}$, des écarts plus importants sont observés. Dans ce cas, les résultats obtenus avec ABAQUS/Explicit et Fluent sont un peu plus proches des résultats expérimentaux que ceux obtenus avec Impact++. Ceci est lié au fait que les hypothèses à la base de la théorie de Wagner sont de moins en moins valides lorsque l'angle de pénétration augmente. Des simulations considérant un cône de $30^{\circ}$ ont également été réalisées par différents auteurs. Battistin et Iafrati (2003) ont obtenu pour valeur de l'effort adimensionnalisé $f_{c}\left(30^{\circ}\right)=4.71$ en utilisant un modèle éléments de frontière basé sur la théorie du potentiel (mais sans aucune linéarisation). Kleefsman et al. (2005) ont quant à eux à l'aide de simulations basées sur la méthode VOF obtenu une valeur de $f_{\mathcal{C}}\left(30^{\circ}\right)$ d'environ 5.4, ce qui semble relativement élevé par rapport aux essais et aux résultats de nos simulations et de ceux de Battistin et Iafrati (2003). 


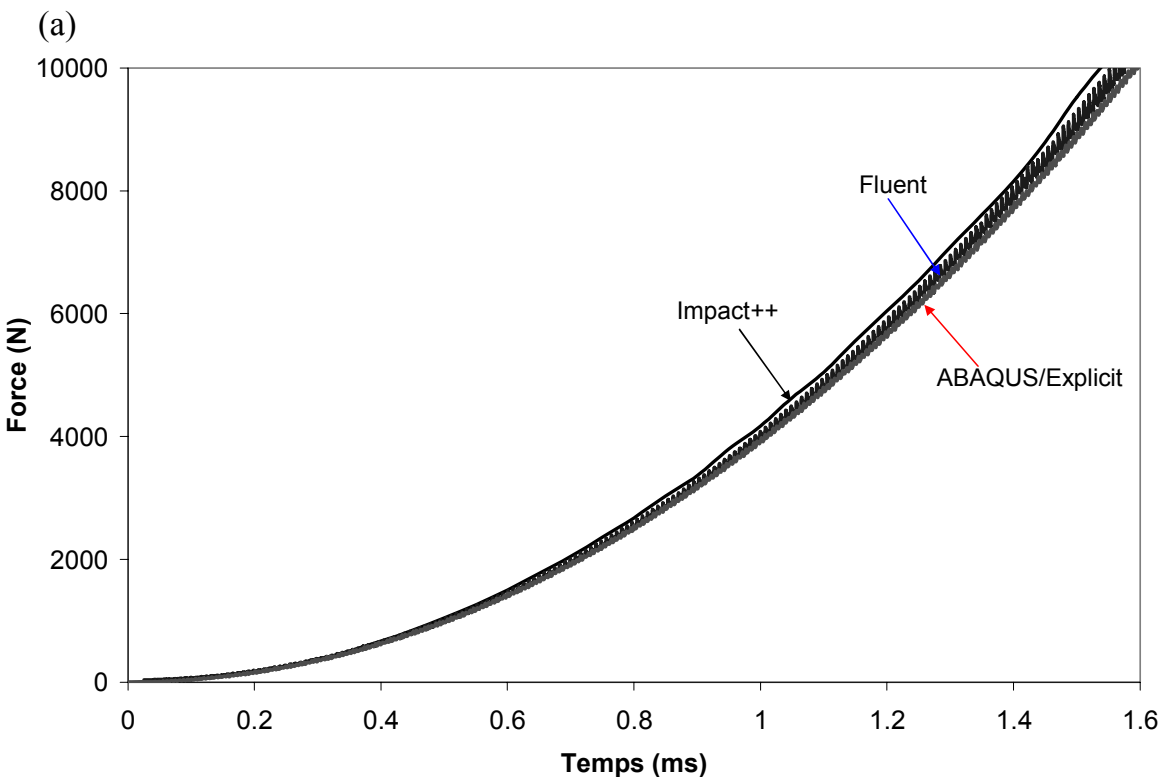

(b)

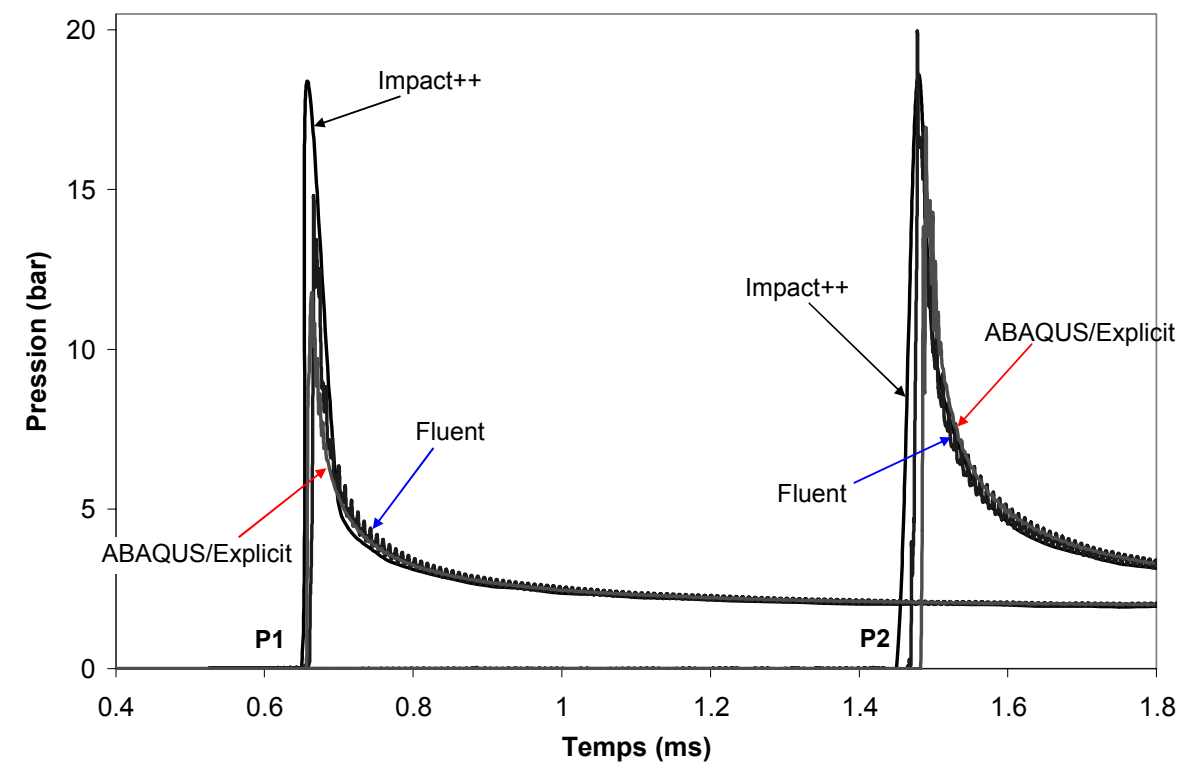

Figure 11. Impact hydrodynamique d'un cône rigide avec un angle de pénétration de $6^{\circ}$. La vitesse d'impact est égale à $5 \mathrm{~m} / \mathrm{s}$. (a) Effort hydrodynamique total, (b) pression au niveau des capteurs de pression (figure 8) 


\begin{tabular}{|c|c|c|}
\cline { 2 - 3 } \multicolumn{1}{c|}{} & P1 (bar) & P2 (bar) \\
\hline Impact++ & 18.35 & 18.35 \\
\hline ABAQUS/Explicit & 11.76 & 16.81 \\
\hline Fluent & 14.81 & 19.96 \\
\hline
\end{tabular}

Tableau 1. Valeurs maximales de la pression au niveau des points $P 1$ et $P 2$ (figure 8) lors de l'impact d'un cône avec un angle de pénétration de $6^{\circ}$ et une vitesse de $5 \mathrm{~m} / \mathrm{s}$

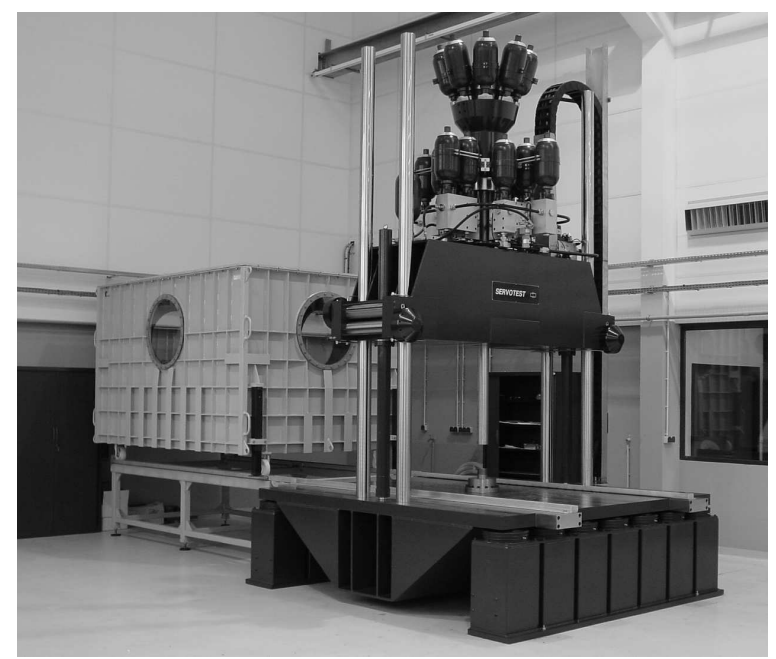

Figure 12. Machine hydraulique pour essais d'impact. Lors des essais d'impact sur l'eau, le bassin (hauteur à mi hublot 1,1 m, longueur $3 \mathrm{~m}$, largeur $2 \mathrm{~m}$ ), visible en arrière-plan, est placé sous la machine

\begin{tabular}{|c|c|c|c|c|}
\hline$\beta\left(^{\circ}\right)$ & ABAQUS/Explicit & Fluent & Impact++ & Essais \\
\hline 7 & 7.18 & 7.2 & 7.68 & 6.79 \\
\hline 15 & 6.25 & 6.26 & 6.77 & 6.18 \\
\hline 30 & 4.66 & 4.68 & 5.26 & 4.75 \\
\hline
\end{tabular}

Tableau 2. Résultats expérimentaux et numériques pour l'impact d'un cône rigide. L'effort adimensionnalisé [27] est donné pour trois valeurs de l'angle de pénétration $\beta$. L'incertitude relative aux résultats expérimentaux est d'environ $10 \%$ 


\subsection{Cylindre}

La figure 13 montre l'évolution temporelle de l'effort hydrodynamique lors de l'impact d'un cylindre circulaire à vitesse constante. Nous voyons que dans ce cas, avec Impact++ et ABAQUS/Explicit, l'effort augmente de manière quasi instantanée au moment de l'impact $(t=0)$, puis décroît ensuite. Ce comportement est également prédit par différents modèles analytiques basés sur la théorie de Wagner (Cointe et Armand, 1987), ainsi que par les simulations numériques réalisées par Battistin et Iafrati (2003) que nous avons déjà évoquées dans la section précédente. On observe que ces derniers résultats, ainsi que ceux d'ABAQUS/Explicit, sont en très bon accord avec les résultats expérimentaux tirés de (Cointe et Armand, 1987). De son côté, Fluent prévoit une augmentation plus progressive de l'effort au moment de l'impact et la valeur maximale atteinte est moins importante. Ceci est lié au principe même de la méthode VOF qui est utilisée par Fluent. En effet, dans cette dernière, la position spatiale des différentes phases est représentée au travers de la fraction volumique au niveau de chaque cellule. Ainsi, il n'y a plus à proprement parler d'interface entre les phases, mais plutôt une zone de transition où la fraction volumique varie progressivement de 0 à 1 . L'épaisseur de cette zone est généralement celle de 3 ou 4 cellules. Cet étalement de la surface libre a pour conséquence de rendre l'impact du cylindre plus «doux » par rapport aux autres modèles.

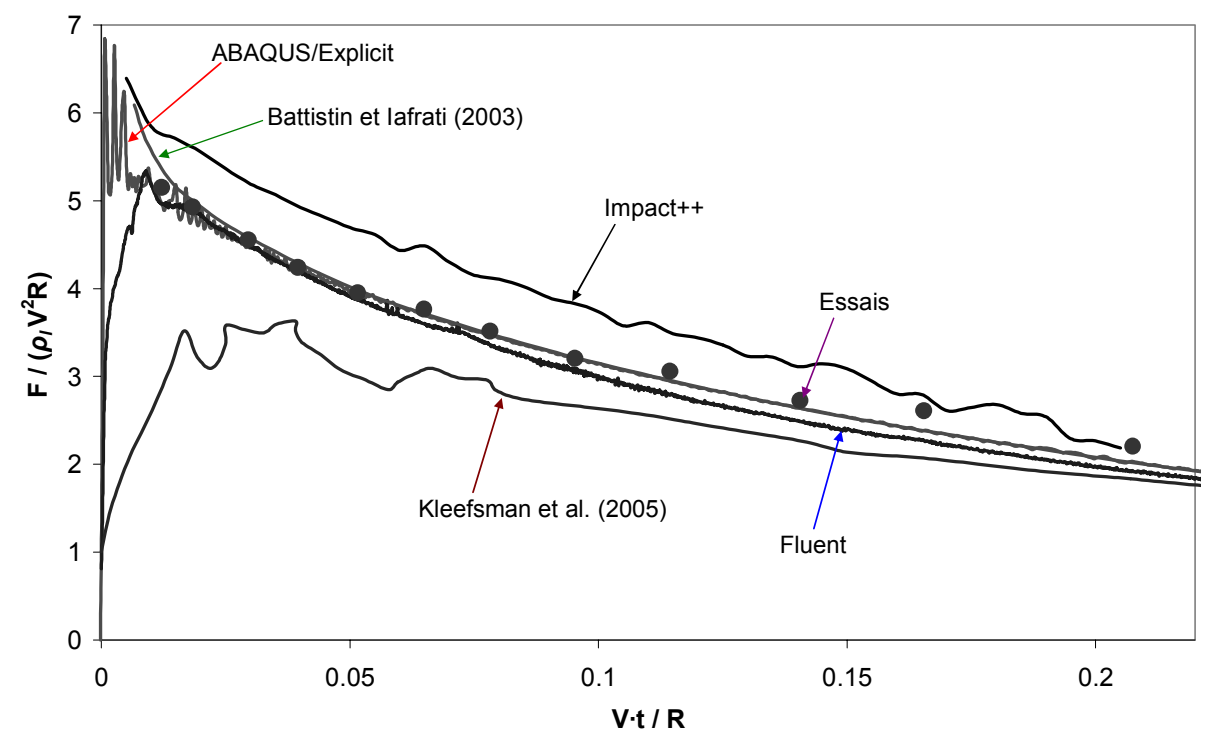

Figure 13. Effort hydrodynamique lors de l'impact d'un cylindre de rayon $R=0.1 \mathrm{~m}$ pour une vitesse d'impact $V=10 \mathrm{~m} / \mathrm{s}$. Les courbes ont été adimensionnalisées pour être comparées aux résultats expérimentaux provenant de (Cointe et Armand, 1987) et aux résultats numériques présentés dans (Kleefsman et al., 2005) et (Battistin et Iafrati, 2003) 
La figure 13 montre également une courbe d'effort obtenue par Kleefsman et al. (2005). Ces auteurs utilisent une méthode VOF originale qu'ils ont implantée dans le code de calcul ComFLOW. Comme nous le voyons, ces simulations prévoient également, et de manière plus marquée que Fluent, une augmentation plus lente de l'effort lors de la phase initiale de l'impact. Cela nous amène à penser que ce problème n'est pas spécifiquement lié à un code donné, mais est sans doute rencontré par de nombreux logiciels basés sur la méthode VOF. La courbe d'effort obtenue avec Fluent rejoint ensuite les résultats d'ABAQUS/Explicit et les résultats expérimentaux. Les simulations réalisées par Kleefsman et al. (2005) semblent quant à elles sous-estimer la valeur de l'effort sur toute la durée. Impact++ fournit une estimation assez correcte de l'effort hydrodynamique, même si la différence avec les résultats d'essais est un peu plus importante.

\section{Conclusions et perspectives}

Dans ce travail, des simulations numériques d'impacts hydrodynamiques ont été réalisées afin d'évaluer les capacités de différents modèles numériques à traiter ce problème et plus particulièrement à prévoir le chargement que subit la structure impactante. Il est à noter que les modèles considérés sont, de part leur formulation, très différents les uns des autres. Les résultats obtenus ont été comparés à des résultats analytiques et expérimentaux. Globalement, une bonne concordance des résultats a été observée. Il est intéressant de noter que des codes commerciaux généralistes (Fluent et ABAQUS) semblent adaptés pour traiter ce type de problème, certes au prix de temps de calcul pouvant être assez importants. Le code Impact++ qui est basé sur la théorie de Wagner a tendance à surestimer légèrement les efforts hydrodynamiques. Néanmoins, un avantage de cet outil est de nécessiter des temps de calcul moins importants. Nous voudrions également indiquer qu'il est sans doute possible d'améliorer les performances du code Impact++. Actuellement la résolution du problème potentiel linéarisé est basée sur la méthode des éléments finis. Or, ce type de problème peut également être traité en utilisant la méthode des éléments de frontière. Dans ce cas, il n'est pas nécessaire de mailler tout le domaine fluide, mais uniquement la surface supérieure, permettant une réduction des temps de calcul. Des améliorations sont sans doute également possibles concernant la précision des résultats. Comme nous l'avons indiqué, dans le cadre de la théorie de Wagner, pour calculer les chargements hydrodynamiques, il est nécessaire d'introduire des termes correctifs pour supprimer la singularité qui apparait au bord de la surface mouillée. Dans le code Impact++, la pression hydrodynamique est calculée en utilisant une formulation s'appuyant sur les travaux de Zhao et Faltinsen (1993). Il existe néanmoins d'autres formulations, voir par exemple (Cointe, 1989) et (Korobkin, 2004). Ce dernier propose en particulier un modèle appelé MLM (modèle de Logvinovich modifié). Dans ce cas, la pression hydrodynamique est déterminée uniquement à partir du potentiel des vitesses calculé à l'aide de la théorie de Wagner, mais des termes correctifs tenant compte de la géométrie réelle du corps 
impactant sont introduits. Ce modèle semble avoir de très bonnes capacités concernant la prédiction des efforts hydrodynamiques, (Malenica, 2007 ; Malleron, 2009). L'utilisation de ce modèle dans le cadre d'Impact++ pourrait améliorer la précision des résultats.

Dans cet article, nous nous sommes limités à l'impact de solides rigides. Des travaux concernant l'impact de structures flexibles ont également été initiés. L'impact de solides tridimensionnels devra être traité dans l'avenir. Notons à ce sujet que le développement de modèles numériques 3D basés sur la théorie de Wagner reste un problème largement ouvert, (Takagi, 2004 ; Gazzola, 2008).

\section{Remerciements}

Nous remercions Aboulghit El Malki Alaoui pour la réalisation des essais d'impact de cônes. Ces essais bénéficient du soutien financier de la Mission pour la Recherche et l'Innovation Scientifique de la DGA.

\section{Bibliographie}

Aquelet N., Souli M., Olovson L., "Euler-Lagrange coupling with damping effects: Application to slamming problems", Computer Methods in Applied Mechanics and Engineering, vol. 195, 2006, p. 110-132.

Azcueta R., Computation of turbulent free-surface flows around ships and floating bodies, Thèse de Doctorat, Université d'Hambourg, 2001.

Battistin D., Iafrati A., "Hydrodynamic loads during water entry of two-dimensional and axisymetric bodies", Journal of Fluids and Structures, vol. 17, ${ }^{\circ}$ 5, 2003, p. 643-664.

Belytschko T., Liu W.K., Moran B., Nonlinear finite elements for continua and structures, Chicester, John Wiley \& Sons, 2000.

Cointe R., "Two-dimensional water-solid impact", Journal of Offshore Mechanics and Arctic Engineering, vol. 111, 1989, p. 109-114.

Cointe R., Armand J.-L., "Hydrodynamic impact analysis of a cylinder", Journal of Offshore Mechanics and Arctic Engineering, vol. 109, 1987, p. 237-243.

Colicchio G., Greco M., Faltinsen O.M., “A BEM-level set domain-decomposition strategy for non-linear and fragmented interfacial flows", International Journal for Numerical Methods in Engineering, vol. 67, 2006, p. 1385-1419.

Colicchio G., Landrini M., Chaplin J.R., "Levet-Set computations of free surface rotational flows", Journal of Fluids Engineering, vol. 127, n 6, 2005, p. 1111-1121.

Constantinescu A., Fuiorea I., Nême A., Bertram V., Salas M., "Hydro-elastic numeric analysis of a wedge-shaped shell structure impacting a water surface", Latin American Applied Research, vol. 38, $\mathrm{n}^{\circ}$ 1, 2008, p. 35-43. 
Constantinescu A., Modélisation 2D de l'impact d'une structure sur l'eau. Initiation de l'endommagement, Thèse de doctorat, Université de Bretagne Occidentale, 2006.

Donguy B., Etude de l'interaction fluide-structure lors de l'impact hydrodynamique, Thèse de doctorat, Ecole Centrale de Nantes, 2002.

Faltinsen O.M., "Water entry of a wedge with finite deadrise angle", Journal of Ship Research, vol. 46, ${ }^{\circ} 1,2002$, p. 39-51.

Faltinsen O.M., Hydrodynamics of High-Speed Marine Vehicles, Cambridge, Cambridge University Press, 2005.

Federico F., Amoruso A., "Impact between fluids and solids. Comparison between analytical and FEA results", International Journal of Impact Engineering, vol. 36, 2009, p. 154-164.

Ferziger J.H., Perić M., Computational Methods for Fluids Dynamics, $3^{\text {rd }}$ Edition, Berlin, Springer, 2002.

Ganesan S., Matthies G., Tobiska L., "On spurious velocities in incompressible flow problems with interfaces", Computers Methods in Applied Mechanics and Engineering, vol. 196, 2007, p. 1193-1202.

Gazzola T., Contribution aux problèmes d'impacts non-linéaires : le problème de Wagner couplé, Thèse de doctorat, Ecole Centrale de Paris, 2007.

Gonzáles D., Cueta E., Chinesta F., Doblaré M., “A natural element updated Lagrangian strategy for free-surface fluid dynamics", Journal of Computational Physics, vol. 223, $\mathrm{n}^{\circ} 1,2007$, p. 127-150.

Hirt C.W., Nichols B.D., "Volume of fluid (VOF) method for the dynamics of free boundaries", Journal of Computational Physics, vol. 39, n 1, 1981, p. 201-225.

Iafrati A., Carcaterra A., Ciappi E., Campana E.F., "Hydroelastic analysis of a simple oscillator impacting the free surface", Journal of Ship Research, vol. 44, n 4, 2000, p. 278-289.

Idelson S.R., Onate E., Del Pin F., "A Lagrangian meshless finite element method applied to fluid-structure interaction problems", Computers and Structures, vol. 81, n 8-11, 2003, p. 583-593.

Khabakhpasheva T.I., "Fluid-structure interaction during the impact of a cylindrical shell on a thin layer of water", Journal of Fluids and Structures, vol. 25, n 3, 2009, p. 431-444.

Kim B., Shin Y.S., "An efficient numerical method for the solution of two-dimensional hydrodynamic impact problems", Proceedings of the thirteen International Offshore and Polar Engineering Conference, Honolulu, Hawaii, USA, may 25-30, 2003.

Kleefsman K.M.T., Fekken G., Veldman A.E.P., Iwanowski B., Buchner B., “A Volume-ofFluid based simulation method for wave impact problems", Journal of Computational Physics, vol. 206, 2005, p. 363-393.

Korobkin A., "Analytical models of water impact", European Journal of Applied Mathematics, vol. 15, 2004, p. 821-838.

Korobkin A., "The entry of an elliptical paraboloid into a liquid at variable velocity", Journal of Applied Mathematics and Mechanics, vol. 66, $\mathrm{n}^{\circ} 1,2002$, p. 39-48. 
Leroyer A., Hay A., Visonneau M., «Intéraction écoulement/mouvement par un solveur Navier-Stokes avec adaptation locale de maillage ; application à l'étude du slamming ", Mécaniques et Industries, vol. 8, $\mathrm{n}^{\circ}$ 2, 2007, p. 151-160.

Malenica S., "Hydro structure interactions in seakeeping", International Workshop on Coupled Methods in Numerical Dynamics, Dubrovnik, Croatia, september 19-21, 2007.

Malleron N., Contribution à l'étude des interactions fluide-structure pour l'analyse de l'impact hydrodynamique d'un système de flottabilité d'hélicoptère, Thèse de doctorat, Université d'Aix-Marseille, 2009.

Mei X., Liu Y., Yue D.K.P, "On the water impact of general two-dimensional sections", Applied Ocean Research, vol. 21, 1999, p. 1-15.

Oger G., Doring M., Alessandrini B., Ferrant P., "Two-dimensional SPH simulations of wedge water entries", Journal of Computational Physics, vol. 213, n 2, 2006, p. 803-822.

Peseux B., Gornet L., Donguy B., "Hydrodynamic impact: Numerical and experimental investigations", Journal of Fluids and Structures, vol. 21, n 3, 2005, p. 277-303.

Ribet H., Laborde P., Mahé M., "Numerical modeling of the impact on water of a flexible structure by explicit finite element method - Comparisons with Radioss numerical results and experiments", Aerospace Science and Technology, vol. 3, n 2, 1999, p. 83-91.

Rompteaux A., Vila J.P., "Calcul du tossage avec "smart fluids", un code particulaire compressible ", Actes des $7^{e}$ Journées de l'Hydrodynamique, Marseille, 8-10 mars 1999.

Scolan Y.M., "Hydroelastic behaviour of a conical shell impacting on a quiescent-free surface of an incompressible liquid", Journal of Sound and Vibration, vol. 277, 2004, p. 163-203.

Sun H., Faltinsen O.M., "Water impact of horizontal circular cylinders and cylindrical shells", Applied Ocean Research, vol. 28, 2006, p. 299-311.

Takagi K., "Numerical evaluation of three-dimensional water impact by the displacement potential formulation", Journal of Engineering Mathematics, vol. 48, 2004, p. 339-352.

Van Leer B., "Towards the ultimate conservative difference scheme. Parts III and IV", Journal of Computational Physics, vol. 23, 1977, p. 263-299.

Varyani K.S., Gatiganti R.M., Gerigk M., "Motions and slamming impact on catamaran", Ocean Engineering, vol. 27, 2000, p. 729-747.

von Kármán T., The impact of seaplane floats during landing, NACA Technical Note No. $321,1929$.

Wagner T., The landing of seaplanes, NACA Technical Memorandum No. 622, 1931.

Youngs D.L., "Time-dependant multi-material flow with large fluid distorsion", Numerical Methods for Fluid Dynamics, Morton K.W. et Baines M.J. (ed), Academic press, New York, 1982.

Zhao R., Faltinsen O.M., "Water entry of two-dimensional bodies", Journal of Fluid Mechanics, vol. 246, 1993, p. 593-612.

Received: 26 August 2009

Accepted: 12 July 2010 